\title{
De l'utilisation de la pause silencieuse dans le débat politique télévisé. Le cas de François Hollande
}

Uses of the silent pause in the political televised debates. A study case: François Hollande's discourse

Usos de la pausa silenciosa en el debate político televisado. El caso de François Hollande

Marion Béchet, Marion Sandré, Fabrice Hirsch, Arnaud Richard, Fabrice Marsac et Rudolph Sock

\section{OpenEdition}

\section{Journals}

Édition électronique

URL : https://journals.openedition.org/mots/21460

DOI : $10.4000 /$ mots. 21460

ISSN : 1960-6001

Éditeur

ENS Éditions

\section{Édition imprimée}

Date de publication : 16 décembre 2013

Pagination : 23-38

ISBN : 978-2-84788-527-9

ISSN : 0243-6450

\section{Référence électronique}

Marion Béchet, Marion Sandré, Fabrice Hirsch, Arnaud Richard, Fabrice Marsac et Rudolph Sock, «De I'utilisation de la pause silencieuse dans le débat politique télévisé. Le cas de François Hollande », Mots. Les langages du politique [En ligne], 103 | 2013, mis en ligne le 16 décembre 2015, consulté le 22 avril 2022. URL : http://journals.openedition.org/mots/21460 ; DOI : https://doi.org/10.4000/mots. 21460 


\section{De l'utilisation de la pause silencieuse dans le débat politique télévisé. Le cas de François Hollande}

«Si l'œil reste rieur, la voix se casse au fil des meetings. [...] Alors son débit à la tribune se densifie. Les mots sont ramassés, entrecoupés de longs silences puis à nouveau une rafale explosive, une véritable compression rythmique, la voix saturée prend des harmoniques aigus. » (Le Monde, 4 mai 2012)

Ces remarques au sujet de François Hollande, lues dans une tribune publiée parAndré Manoukian sur le site du Monde, sont révélatrices du fait que la forme du discours est tout aussi importante que le contenu, dans la mesure où la première participe pleinement à la construction d'une image de présidentiable. En outre, ces mêmes phrases sont la preuve de la singularité de l'élocution propre à François Hollande, qui est notamment définie ici par un débit rapide et de longues interruptions.

L'objectif de cet article est justement d'étudier le rythme dans le discours de François Hollande, en se focalisant plus particulièrement sur l'utilisation qu'il fait des pauses, et ce afin de comprendre les spécificités de sa parole. Notre hypothèse est que le placement des pauses est loin d'être anodin, dans la mesure où elles contribuent à la structuration et à la mise en lumière de certaines parties du discours. Ces interruptions rythmiques seront étudiées à travers deux débats politiques télévisés en face-à-face.

\footnotetext{
Institut de Phonétique de Strasbourg, LiLPa - Parole et cognition marionbechet@yahoo.fr Université Paul Valéry Montpellier 3, Praxiling (UMR 5267 CNRS) marion.sandre@univ-montp3.fr Université Paul Valéry Montpellier 3, Praxiling (UMR 5267 CNRS) fabrice.hirsch@univ-montp3.fr Université Paul Valéry Montpellier 3, Praxiling (UMR 5267 CNRS) arnaud.richard@gmail.com Université d’Opole, Pologne fmarsac@uni.opole.pl Université de Strasbourg, Institut de Phonétique de Strasbourg sock@unistra.fr
} 


\section{Études antérieures sur la pause}

Le temps est un élément fondamental de la communication orale qui permet un accès progressif à l'information discursive ainsi que la mise en évidence de l'argumentation en parole spontanée. Le temps peut aussi devenir un véritable enjeu dans les messages orientés essentiellement vers l'auditeur, qu'on veuille le disputer à son adversaire dans des échanges directs, ou qu'on veuille l'exploiter au maximum dans des échanges indirects. Dans ces cas précis, la gestion des pauses peut se révéler stratégique.

\section{Description de la pause}

Lorsqu'on travaille sur du discours dialogué, qui se présente sous forme de tours de parole, on peut distinguer deux types de pauses : les pauses intra-tours, qui sont intimement liées à la production du discours, et les pauses inter-tours, lesquelles se produisent lors du changement de locuteurs. Ces dernières correspondent à la passation de la parole entre les différents participants au dialogue. Ce temps de passation, qui dure en moyenne quelques dixièmes de secondes (et qui est sujet à variation interculturelle) ${ }^{1}$, participe au principe d'alternance des tours : il respecte la «minimisation des silences et des chevauchements» (minimization of gap and overlap, Sacks et al., 1974, p. 714). Lorsqu'il est plus long, un silence prolongé est alors perceptible entre deux tours ( $g a p$ ), ce qui est considéré comme un « raté du système des tours» (Kerbrat-Orecchioni, 1990, p. 172-173), dans le sens où il transgresse la règle susmentionnée. Dans le genre de discours qui nous intéresse, les pauses inter-tours sont brèves et très fréquemment remplacées par un chevauchement de parole (Sandré, 2010). Ces phénomènes relèvent de la gestion, par les participants, de la passation de la parole dans l'interaction et ne renvoient pas aux mêmes mécanismes de production que les pauses intra-tours. Afin de traiter la manière dont un locuteur utilise les pauses dans son propre discours, nous ne traiterons pas les pauses intertours et focaliserons l'analyse sur les pauses intra-tours. Ces dernières interviennent à l'intérieur du discours d'un locuteur : elles participent activement à la production du discours. Plusieurs études ont tâché de quantifier leur durée. Ainsi, les résultats obtenus par François Grosjean et Alain Deschamps (1975) ont par exemple montré que les pauses vides duraient $520 \mathrm{~ms}$ (millisecondes) en moyenne pour un locuteur en train de s'exprimer dans un entretien. En outre, Danielle Duez (2003) a notamment relevé que les pauses produites par Lionel Jospin dans le débat qui l'opposait à Jacques Chirac, en 1995, étaient généralement comprises entre $147 \mathrm{~ms}$ et $635 \mathrm{~ms}$.

1. Voir Kerbrat-Orecchioni, 1994, p. 24-25. 
D'un point de vue phonétique, une pause vide correspond à une interruption du flux de parole qui se caractérise par une amplitude non significative sur le signal acoustique. Ces silences coïncident, dans certains cas, avec une prise de respiration qui est nécessaire lors de la production de la parole. Comme le souligne Maria Candea (2000), ces brèves inspirations, de même que les pauses sans prise de souffle, n'interviennent pas de manière aléatoire dans le discours d'un locuteur, dans la mesure où c'est ce dernier qui, hors cas de pathologie, choisit l'emplacement de ses prises de souffle. Ainsi, à l'exception de certains cas qui seront évoqués plus loin (voir infra, «Fonction des pauses silencieuses »), un locuteur aura normalement tendance à privilégier les frontières phono-syntaxiques pour réaliser des pauses plutôt que de couper la progression de son énoncé à un endroit inapproprié comme, par exemple, à l'intérieur d'un mot (Zellner, 1998). Cela s'explique par le fait que la pause constitue un élément fondamental de la structuration temporelle de la parole, étant donné qu'elle correspond au temps marqué pour l'organisation de l'énoncé, pour l'hésitation ainsi que pour l'accès à l'information lexicale. En outre, elle joue un rôle important dans la perception des unités linguistiques ainsi que dans l'interprétation des situations. L'analyse de la pause est donc d'un intérêt évident pour la connaissance de l'organisation temporelle de la parole et, d'une manière plus générale, pour l'analyse du discours.

\section{Fonction des pauses silencieuses}

L'interruption momentanée du signal de parole peut avoir une valeur particulière au niveau linguistique, raison pour laquelle les pauses silencieuses ne contiennent pas systématiquement de prise de souffle. Le silence, dans un énoncé, permet en effet à un locuteur de planifier ce qui va être dit et de structurer un énoncé, ce qui fait d'ailleurs dire à Frieda Goldman-Eisler (1968) qu'il est essentiel à la production d'un énoncé. La pause s'avère également utile pour l'interlocuteur, dans la mesure où elle donne le temps à ce dernier de mieux intégrer le message émis par le sujet parlant (Dister, 2008). Plusieurs linguistes ont ainsi proposé une description des différentes fonctions que le silence peut prendre.

On peut ainsi citer Raimund H. Drommell (1980) qui distinguait les pauses syntaxiques et non syntaxiques, ou encore Donald S. Boomer et Allen T. Dittman (1962) qui proposaient de différencier les pauses démarcatives et les pauses non démarcatives, ces dernières étant considérées comme des silences d'hésitation. La différence majeure entre ces deux types de silence réside dans le fait que le locuteur place les premiers de manière volontaire et à des moments précis dans son énoncé, alors que les secondes sont réalisées à des emplacements indépendants de la volonté du sujet en train de produire l'énoncé. De manière plus précise, les pauses démarcatives participent à l'organisation 
sémantique et syntaxique du discours dans la mesure où elles permettent de regrouper les mots fonctionnant ensemble et qui forment des unités de sens. Les pauses d'hésitation peuvent être définies comme des disfluences, voire comme la preuve d'un travail de reformulation effectué par le locuteur (Morel, Danon-Boileau, 1998). Ces silences dus à des hésitations sont susceptibles d'apparaître à n'importe quel moment sur le signal de parole dans le sens où ils peuvent couper en deux un groupe de sens.

Plusieurs études ont tenté de quantifier ces différents types de pauses sans qu'un consensus ait pu être trouvé. Si, pour Donald Boomer (1965), l'hésitation est d'une durée plus longue que la démarcation, Danielle Duez (1995) n'a pas relevé de différence significative entre ces deux catégories de pause d'un point de vue temporel.

Gaëlle Ferré (2003, 2004), qui a travaillé sur un corpus de parole spontanée en anglais, a distingué quant à elle trois pauses différentes : les pauses d'hésitation, les pauses de focalisation et les pauses démarcatives. Les premières seraient notamment repérables par la présence d'autres marques d'hésitation comme un « euh » et par un débit plus lent avant la pause puis plus rapide après celle-ci. D'un point de vue interactionnel, ces silences sont un moyen offert au locuteur de gagner du temps et trouver ses mots (Sacks et al., 1974). Quant aux silences de focalisation, ils sont utilisés pour donner du relief à l'élément du discours placé juste après. Ces pauses sont généralement précédées par une suite sonore prononcée dans un débit rapide et suivies d'une autre suite sonore prononcée à une vitesse d'élocution plus lente. Enfin, les pauses de démarcation, dont il a déjà été question, sont celles qui ont pour objet d'établir des frontières entre les différents groupes intono-syntaxiques. Dans certains cas, notamment lorsque le locuteur a peur d'être interrompu par son interlocuteur, il se peut que cette pause démarcative soit « décalée vers la droite », autrement dit qu'elle apparaisse à l'intérieur du groupe de sens suivant. Cette stratégie, qui consiste à déplacer la pause sans pour autant mettre en valeur un élément du groupe intono-syntaxique dans lequel elle se trouve, vise principalement à conserver la parole. En effet, la pause démarcative, qui se situe à la fin d'une unité de sens, peut être interprétée par les interlocuteurs comme la fin d'un tour de parole, et donc comme un moment propice pour l'alternance des locuteurs : une "place de transition pertinente (transition relevance place, Sacks et al., 1974). Le fait de décaler la pause démarcative permet de signifier que le tour n'est pas achevé, et le locuteur peut ainsi poursuivre son propos sans se faire interrompre. Notons que ces silences, qui désobéissent aux règles syntaxiques et qui sont souvent situés après un mot-outil, ne peuvent être assimilés à une hésitation dans la mesure où aucun indice (gestuel ou auditif) n'est présent pour l'attester. Ferré (2004) ajoute que ces pauses sont propres à la parole spontanée et explique qu'elles constituent une stratégie conversationnelle permettant de conserver son tour de parole. 
Ces différentes catégories de pauses sont soumises à une certaine variabilité intra- et inter-locuteurs. De manière générale, l'utilisation de la pause n'est pas la même selon les genres du discours : la production du discours est très différente s'il s'agit d'un discours monologal ou dialogal, formel ou informel, direct ou médiatisé (Sandré, 2013). Duez (2003) a ainsi montré que le nombre de pauses n'est pas le même selon qu'un homme politique s'exprime dans une conversation informelle ou dans un entretien politique : les hésitations sont plus nombreuses dans le premier cas alors que la durée des pauses est plus longue dans le second.

\section{Méthodologie}

\section{Le corpus}

Pour mener cette étude, deux débats d'entre-deux-tours ont été retenus et ont fait l'objet d'un enregistrement vidéo (format .mp4) et audio (.wav). Le premier est celui de l'élection présidentielle française de 2012 qui s'est déroulé le 2 mai 2012 et qui opposait François Hollande à Nicolas Sarkozy. Il était animé par David Pujadas et Laurence Ferrari. Le second est atypique (Richard, Sandré, 2013) puisqu'il s'agit du débat de l'entre-deux tours des primaires citoyennes, qui visaient à départager des candidats socialistes et radicaux de gauche en vue de l'élection présidentielle à venir. Le second tour de cette élection, qui s'est déroulé le 12 octobre 2011, opposait deux personnalités appartenant au même parti, à savoir Martine Aubry et François Hollande. Il était organisé par David Pujadas, l'animateur représentant la chaîne de diffusion France 2, et ponctuellement par trois journalistes : Fabien Namias - responsable du service politique de France 2, Patrick Cohen - journaliste à France Inter - et Françoise Fressoz - éditorialiste au journal Le Monde.

Ces deux débats ont l'avantage de présenter le locuteur François Hollande dans une même situation de parole. Certes, un certain nombre de différences opposent les deux échanges : le premier débat appartient à la campagne présidentielle officielle, le second est organisé par le Parti socialiste. Martine Aubry est une partenaire politique autant qu'une adversaire, alors que Nicolas Sarkozy appartient à un autre parti. La première est une femme, le second un homme... Nous retiendrons plutôt les convergences : les deux sont des débats télévisés en face-à-face. Le discours de François Hollande, comme tout discours entrant dans l'exercice de la profession politique, est préparé et répété, mais l'interaction l'oblige aussi à improviser face au discours de l'adversaire. Le dialogue est donc garant d'une certaine spontanéité. 


\section{Analyses phonétiques}

L'analyse prosodique a été effectuée sur 45 minutes de temps de parole chez François Hollande dans chacun des débats décrits ci-dessus (soit $1 \mathrm{~h} 30$ de transcription) ${ }^{2}$.

Afin de pouvoir obtenir des données sur le rythme de parole employé par le futur président de la République, une transcription fine de l'intervention orale a été réalisée à l'aide du logiciel Praat. Ce travail initial consistait à étiqueter l'ensemble des énoncés produits par François Hollande et à repérer, sur le signal acoustique, les pauses que ce dernier réalisait. Comme pour la plupart des autres travaux menés sur le sujet (voir par exemple Candea, 2000), seules les pauses égales ou supérieures à $200 \mathrm{~ms}$ ont été retenues, étant donné notamment qu'il s'agit du seuil à partir duquel une pause est susceptible d'être correctement décelée par des auditeurs dans un énoncé.

Ce travail préalable a ainsi permis le relevé des suites de phonèmes situées entre deux silences, que l'on appellera désormais "séquences sonores», et des pauses. Signalons enfin qu'un comptage manuel du nombre de syllabes par séquence sonore a été effectué, une fois étiqueté l'ensemble des deux débats.

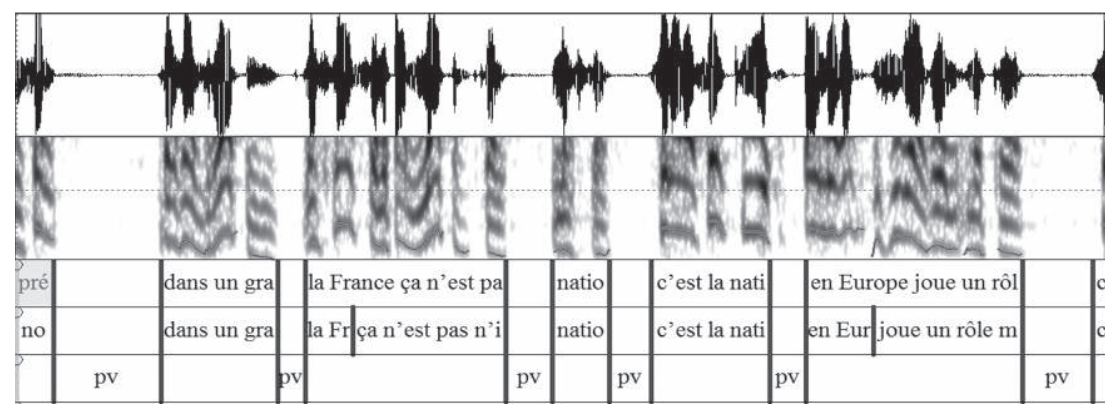

Figure 1 : Exemple d'étiquetage d'un signal de parole de François Hollande*

*Exemple d'étiquetage du signal sur « Nous sommes / dans un grand pays / La France, ça n'est pas n'importe quelle / nation / C'est la nation qui / en Europe joue un rôle moteur / C'est... » (pv = pause vide).

Lorsque ces premières étapes ont été terminées, une quantification des paramètres suivants a été réalisée (Béchet et al., à paraître) :

- le nombre de syllabes moyen par séquence sonore;

- la vitesse d'élocution (en nombre de syllabes/seconde);

2. Sur les 4 h 40 que durent les deux débats ( 1 h 50 pour le débat de la primaire et 2 h 50 pour le débat de la présidentielle). 
- la durée moyenne des séquences sonores;

- la durée moyenne des pauses;

- le ratio temps de parole / temps de pause.

Par la suite, une distinction a été établie entre les pauses d'hésitation, de démarcation et de focalisation. Afin de catégoriser les différents types de silence, nous avons considéré qu'une pause démarcative était caractérisée par un silence séparant deux séquences sonores différentes de manière logique, c'est-à-dire sans rompre un groupe intono-syntaxique. Ont été définis comme pauses d'hésitation les silences situés à proximité d'une pause pleine de type «euh ». Enfin, les pauses de focalisation sont celles mettant en évidence un élément précis du discours. Bien que la classification des différentes pauses fût réalisée à partir de critères définis à l'avance, il nous a semblé que cette démarche était relativement subjective. De ce fait, un jury de deux experts a eu pour tâche de catégoriser les pauses présentes dans les énoncés de François Hollande pour chaque débat. Notons enfin que des mesures statistiques (Anova One Way) ont été menées sur nos données afin de vérifier le degré de significativité des résultats.

\section{Analyse linguistique}

L'analyse du discours a été réalisée grâce aux données audiovisuelles : la transcription fait apparaître les données paraverbales mises en évidence par l'approche phonétique, ainsi que d'autres données (paraverbales, verbales et non verbales) qui permettent d'interpréter le discours produit. L'analyse considère ainsi l'ensemble du discours, son contexte de production (le genre du discours) et l'interaction (prise en compte des phénomènes interlocutoires entre les participants et de la relation interpersonnelle).

L'analyse du discours en interaction considère que le discours est coconstruit : les pauses qui figurent dans le discours d'un des participants sont négociées avec les autres interactants. L'analyse doit englober l'ensemble des paramètres permettant d'interpréter la production et la façon d'utiliser les pauses dans le discours.

\section{Résultats}

\section{Analyse rythmique globale}

L'étude rythmique réalisée sur la parole de François Hollande a notamment permis de révéler une première tendance montrant que le candidat socialiste s'exprimait dans un débit légèrement plus rapide face à Martine Aubry, où sa vitesse d'articulation globale a été quantifiée à 6,20 syllabes/seconde contre 5,85 syllabes/seconde lorsqu'il est opposé à Nicolas Sarkozy (voir figure 2). 
Ce résultat peut notamment s'expliquer par le fait que la durée moyenne des séquences sonores était plus élevée ( $p<0,000)$ chez François Hollande quand il était opposé au président sortant, ce paramètre étant alors mesuré à 1412 ms en moyenne (écart-type $=896 \mathrm{~ms}$ ) que face à Martine Aubry, où la durée moyenne des séquences sonores était de 1135 ms (écart-type : $681 \mathrm{~ms}$ ). Il est intéressant d'ajouter à cela que le nombre moyen de syllabes par séquence sonore est moins élevé ( $p<0,000$ ) lorsque le candidat s'exprime dans la primaire socialiste (7,04 syllabes/seconde avec un écart-type à 4,66) que dans le débat présidentiel (8,44 syllabes/seconde avec un écart-type à 5,48). Si les deux débats sont importants dans la carrière politique de François Hollande, celui qui l'oppose au président sortant est particulièrement crucial et solennel : c'est le dernier événement de la campagne présidentielle. On peut donc penser que son discours est plus maîtrisé dans ce débat, ce qui implique un débit plus lent.
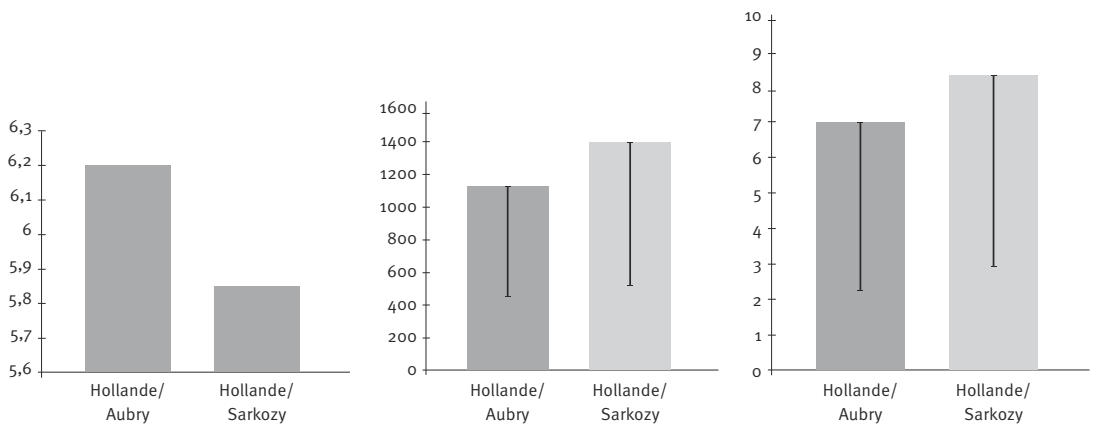

Figures 2-3-4

Figure 2 : Comparaison de la vitesse d'articulation chez François Hollande face à Martine Aubry et face à Nicolas Sarkozy (en nombre de syllabes/seconde).

Figure 3 : Durée des séquences sonores chez François Hollande face à Martine Aubry et face à Nicolas Sarkozy (en millisecondes).

Figure 4 : Nombre de syllabes/séquence sonore chez François Hollande face à Martine Aubry et face à Nicolas Sarkozy.

Le ratio temps de parole / pause est également comparable dans les deux débats, ce dernier étant de $77 \%$ de temps de parole contre $23 \%$ de temps de pause chez François Hollande lorsqu'il est opposé à Martine Aubry vs $76 \%$ de phonation et $24 \%$ de silence lorsque ce même candidat est opposé à Nicolas Sarkozy. En termes de valeurs absolues, cela signifie que, sur les 44 minutes d'analyses faites sur la parole de François Hollande dans le débat présidentiel par exemple, la durée totale des pauses vides mises bout à bout s'élève à plus de 11 minutes. Ce résultat souligne bien l'importance de la ou plutôt des pauses dans le discours politique. 


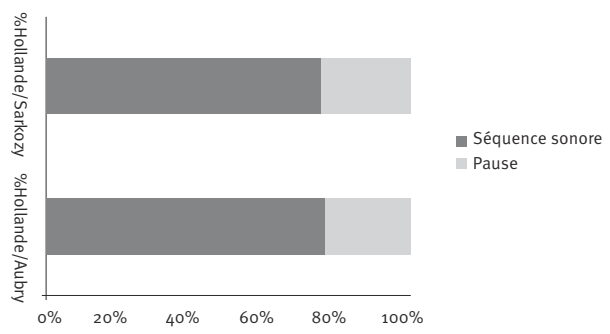

Figure 5. Ratio Temps de parole (séquence sonore) / Temps de pause chez François Hollande face à Martine Aubry et face à Nicolas Sarkozy

\section{Typologie des pauses}

De manière générale, la durée des pauses vides réalisées par François Hollande s'élève à $442 \mathrm{~ms}$ sur l'ensemble du débat présidentiel et à $370 \mathrm{~ms}$ durant le débat de la primaire citoyenne. Cette durée plus longue face à Nicolas Sarkozy irait dans le sens d'une plus grande maîtrise de sa parole lors du face-àface présidentiel. Par ailleurs, signalons que la durée des pauses chez le candidat socialiste dans ces deux émissions est inférieure aux résultats obtenus par Grosjean et Deschamps (1975) et comparable aux observations de Duez (2003) au sujet de Lionel Jospin, ce qui laisse penser qu'il ne s'agit pas du paramètre principal responsable de la singularité rythmique de François Hollande.

Enfin, la forte variabilité que l'on peut relever au niveau des pauses produites dans les deux débats, avec un écart-type à 233 ms lors du face-à-face présidentiel et à 171 ms contre Martine Aubry, laisse penser qu'il n'existe pas qu'une seule sorte de pause.

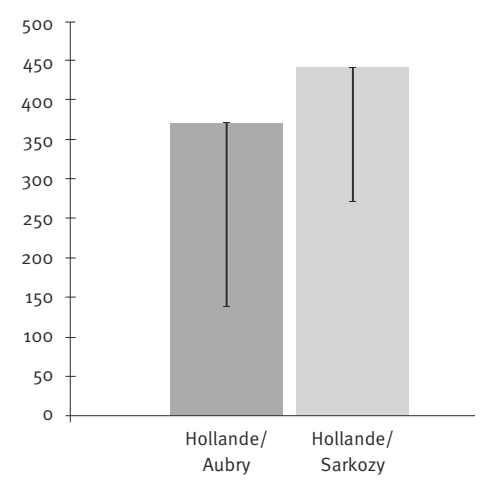

Figure 6 : Durée moyenne des pauses chez François Hollande face à Martine Aubry et face à Nicolas Sarkozy (en millisecondes) 
Pour présenter ces différentes variétés de pauses, nous avons repris les catégories de Gaëlle Ferré (2003), à savoir les pauses d'hésitation, les pauses de focalisation et les pauses démarcatives (ces dernières pouvant être légèrement décalées vers la droite). Dans la transcription, elles sont notées respectivement H, F, D et DD. Les extraits analysés proviennent des débats Aubry-Hollande (noté 2011) et Hollande-Sarkozy (noté 2012), dont les prises de parole sont numérotées (et figurent après la date du débat dans les exemples).

En premier lieu, voici des exemples représentatifs de ces types de pause :

(1)3 nous nous dirons les choses \{559-D $\}$ sans qu'il y ait euh $\{389-\mathrm{H}\}$ quelque chose à cacher à dissimuler $\left(\mathrm{FH}_{2012}\right.$,11)

L'exemple (1) illustre la pause démarcative et la pause d'hésitation. La première sépare deux groupes de sens, plus précisément une proposition principale et une proposition subordonnée. Elle se situe donc naturellement juste avant «sans que». La seconde suit immédiatement le marqueur d'hésitation euh, considéré comme une pause pleine. La présence de cette interjection indique que François Hollande a du mal à trouver ses mots. Cependant, la pause elle-même reste assez brève, et il poursuit rapidement son propos.

L'exemple suivant illustre la pause de focalisation :

(2) > je n'suis pas non plus $<\{246-\mathrm{F}\}$ une gauche molle $/\left(\mathrm{FH}_{2011}, 78\right)$

La pause se situe ici au milieu de l'énoncé, entre le verbe et l'attribut du sujet. Il n'y a donc aucune raison pour que ce soit une pause démarcative. Aucun signe ne permet d’identifier la pause comme une pause d'hésitation (pas de euh, d'allongement vocalique, de répétition de mots, de ralentissement de débit...). En revanche, le syntagme qui suit est important : cette dénomination appartient au discours de Martine Aubry. Celle-ci, lors d'un précédent débat, s'est opposée à la «gauche molle» en même temps qu'elle s'opposait à son adversaire socialiste. Le présentateur David Pujadas demande donc aux deux finalistes de se positionner par rapport à cette catégorisation, que François Hollande se défend de représenter ici4. La pause permet par conséquent de mettre à distance ce syntagme pour affirmer incarner « une gauche sincère et crédible».

Enfin, l'exemple (3) montre ce que peut être une pause démarcative décalée :

(3) > je veux aussi saluer les militants socialistes parce que $<\{248-D D\}$ ils ont organisé/ (FH2011, 64)

3. Conventions de transcription, mises en évidence ici par les guillemets français : les pauses sont notées entre accolades et chronométrées en millisecondes; « $\backslash$ » intonation montante/ descendante; «〉... «» accélération du débit de parole; «BELLE» : accentuation de la syllabe; "(hochement de la tête) “...”" : description du comportement verbal concernant le phénomène circonscrit par les guillemets. Le gras signale la catégorisation de la pause.

4. Voir l'analyse dans Richard, Sandré, 2012, p. 652-653. 
Dans cet énoncé, la pause figure non pas entre la proposition principale et la proposition subordonnée, comme dans l'exemple (1), mais elle se situe juste après la locution conjonctive «parce que». Le fait de s'arrêter ainsi de façon décalée est une technique pour conserver la parole. En effet, si François Hollande s'était arrêté de parler - même pour un court instant - après "socialistes ", les interlocuteurs auraient pu penser qu'il avait achevé son discours. En revanche, la pause après la locution conjonctive ne peut en aucun cas être comprise comme la fin d'un tour. Les pauses démarcatives décalées sont surtout utilisées lorsque le locuteur sent qu'il peut se faire interrompre. En effet, dans ce passage, le présentateur David Pujadas émet de nombreux signes pour reprendre la parole au candidat. Pressé, le candidat socialiste modifie sa façon d'élaborer son discours afin de pouvoir aller au bout de ce qu'il veut dire. Ce type de pause est plus rare.

Les pauses démarcatives sont statistiquement plus importantes $(p<0,000)$ que les pauses de focalisation et d'hésitation. D'un point de vue temporel, on relève que les pauses délimitant les séquences sonores durent en moyenne 532 ms (écart-type : $277 \mathrm{~ms}$ ), alors que les focalisations et les hésitations mesurent respectivement $345 \mathrm{~ms}$ (écart-type : $126 \mathrm{~ms}$ ) et $347 \mathrm{~ms}$ (écart-type : 179 ms). Il est à noter que si ces deux catégories de silence sont temporellement comparables, la durée des pauses d'hésitation semble plus variable que celle des pauses de focalisation.

Signalons également que pour ces trois types, les pauses sont toutes plus longues lorsque François Hollande est confronté à Nicolas Sarkozy : les démarcations, hésitations et focalisations sont en effet respectivement de $582 \mathrm{~ms}$, $360 \mathrm{~ms}$ et $363 \mathrm{~ms}$ dans ce débat et de 482 ms, 334 ms et 327 ms face à Martine Aubry.

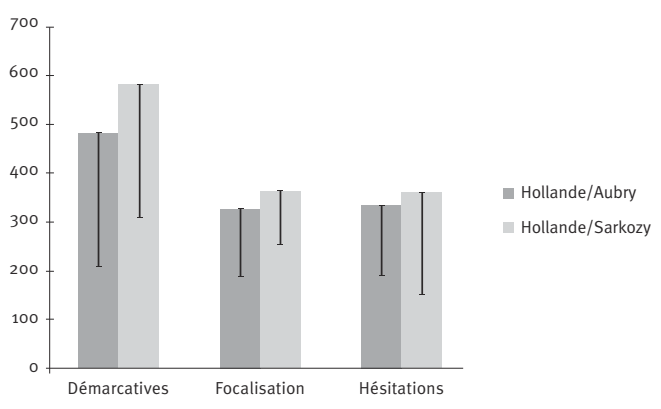

Figure 7. Durée moyenne des pauses démarcatives, de focalisation et d'hésitation chez François Hollande face à Martine Aubry et face à Nicolas Sarkozy*

*Résultats obtenus sur 50 pauses démarcatives, 50 pauses de focalisation et 50 pauses d'hésitation pour chaque débat (soit 300 mesures). 
L'étude de la répartition des pauses produites par François Hollande (fig. 8) révèle, comme attendu, une majorité de pauses démarcatives (58\% des cas). On remarque également que les pauses démarcatives décalées ne constituent que $5 \%$ des interruptions sonores, ce qui laisse penser que le déplacement des pauses est une stratégie utile dans le but, notamment, de ne pas céder la parole, mais elle ne peut être trop fréquemment employée sous peine de destructurer le discours. Enfin, il est possible de constater, sur cette même figure, que le pourcentage relativement important de pauses de focalisation (19\%) et d'hésitation (18\%) pourrait constituer la «marque de fabrique » de la parole de François Hollande.

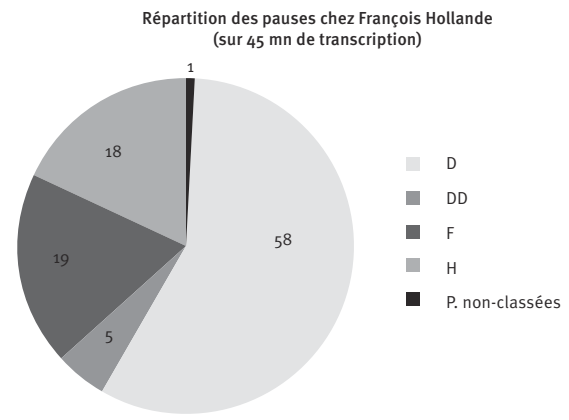

Figure 8. Répartition des pauses démarcatives (D), démarcatives décalées (DD), de focalisation (F), d'hésitation (H) dans la parole de François Hollande

Enfin, signalons la présence d'un certain nombre de pauses (1\%) difficilement classables parmi les trois catégories mentionnées.

\section{Quelques exemples d'emploi des pauses dans le discours politique}

La pause est un phénomène récurrent dans l'élaboration du discours. En ce sens, il est difficile d'isoler des phénomènes. Le plus souvent, on a affaire à un enchevêtrement de différentes pauses et de différentes stratégies discursives. La catégorisation n'est pas toujours simple, raison pour laquelle nous allons nous intéresser à des exemples un peu plus longs pour montrer la dynamique d'utilisation des pauses. L'exemple (4) est tiré du débat Aubry-Hollande, et ce dernier aborde la question du retour à l'équilibre budgétaire de la France en 2017 :

(4) le plus difficile c'est la marche $\{443-\mathrm{D}\}$ vers deux mille treize $\backslash\{910-\mathrm{D}\}$ puisqu'il va falloir passer d'un déficit à peu près euh $\{609-\mathrm{H}\}$ de euh $\{297-\mathrm{H}\}$ quatre virgule six pour cent $/\{583-D\}$ à trois pour cent $\{407-D\}$ en une seule année $\{595-D\}$ rendez vous compte $h\{1141-D\}$ mais j'vais dire pourquoi je suis contre la dette $\backslash\{1049-D\}$ parce que euh $\{305-\mathrm{H}\}$ euh aujourd'hui le premier budget de l'état $\{1063-\mathrm{D}\}$ c'est 
\{276-F\} le paiement deS INtérêts de l'emprunt \{592-F\} pas des REMboursements \{733-F\} deS INtérêts de l'emprunt $\backslash\{523-D\}$ c'est plus important \{396-D $\}>$ que le budget d'l'enseignement scolaire < $(\mathrm{FH} 2011,155)$

Cet extrait est le début de la prise de parole 155 : François Hollande répond ici à une question de David Pujadas. Ayant la parole de plein droit, il prend le temps de développer son discours. Les premières pauses sont démarcatives; s'ensuit une hésitation sur le chiffrage du déficit public (4,6\% du Produit intérieur brut). Deux pauses d'hésitation, accompagnées du marqueur euh, encadrent la préposition de. Ensuite, le candidat socialiste reprend le développement de son argument, en utilisant de nouveau des pauses démarcatives. On peut à ce titre noter une pause plus longue après l'énoncé interpellant l'interlocuteur: «rendez-vous compte ». Il s'agit là véritablement d'une pause entre deux périodes de son discours. Vers la fin de l'extrait, François Hollande utilise plusieurs pauses de focalisation : elles lui permettent de mettre en valeur ses arguments. On les trouve avant les syntagmes «le paiement des intérêts de l'emprunt », "pas des remboursements », " des intérêts de l'emprunt ». Ces trois éléments discursifs sont accentués (ce qu'indiquent les majuscules), le premier est répété : à la fois les pauses, les accentuations et la répétition sont utilisées pour renforcer sa démonstration.

L'exemple (5) est, lui, tiré du face à face Hollande-Sarkozy :

(5) est-ce que je dois vous rappeler $\{511-D\}$ nicolas sarkozy $\{409-D\}$ que vous êtes au pouvoir depuis DIX ans \{526-F\} DIX ans \{701-D $\}$ pas simplement CINQ ans > comme président de la république < \{394-D $\}$ mais vous étiez CINQ ans \{277-D $\}$ ministre euh $\{248-\mathrm{H}\}$ sous jacques chirac dont une partie $\{351-\mathrm{D}\}>$ comme ministre de l'économie et des finances $\backslash(\mathrm{FH} 2012,30)$

Dans ce passage, François Hollande répond à son interlocuteur, qui explique le recul de la France par la réforme des trente-cinq heures menée par le gouvernement socialiste. François Hollande, qui représente ce camp politique, contre-attaque en demandant à son adversaire de prendre ses responsabilités. Il s'agit donc d'un discours polémique, ouvertement critique. La plupart des pauses dans cet extrait sont démarcatives. On peut noter les deux pauses qui encadrent l'apostrophe "nicolas sarkozy » : l'utilisation de cette interpellation nominative est argumentative. En rappelant ainsi son prénom et son nom, le candidat socialiste semble prononcer une sentence juridique et identifier le coupable. Il évite aussi de faire référence à sa fonction et n'emploie pas par exemple «Monsieur le Président »5 pour faire référence à celui qu’il veut affronter comme un adversaire. Les pauses permettent de mettre en valeur la

5. Ce procédé rappelle la joute verbale, restée célèbre, qui a opposé, lors du débat pour les élections présidentielles, le 28 avril 1988, le candidat Jacques Chirac à François Mitterrand, alors président sortant : «Nous sommes deux candidats, à égalité [...] Vous me permettrez donc de vous appeler Monsieur Mitterrand - Mais vous avez tout à fait raison, Monsieur le Premier ministre.» 
structuration du discours (on pourrait à ce titre les interpréter aussi comme des pauses de focalisation). Enfin, on peut noter la pause de focalisation avant le syntagme «dix ans » : ce dernier est mis en valeur par l'accentuation, la pause, la répétition du même syntagme. Ces différents procédés emphatiques contribuent ici aussi à l'argumentation de François Hollande.

Les pauses seules sont difficilement classables. On ne peut les analyser qu'en étroite synergie avec la teneur du discours, les phénomènes d'élaboration du discours (les données paraverbales, les marqueurs d'hésitation, les répétitions lexicales...) et le contexte interactionnel, qui permet de savoir dans quelle situation interlocutoire se trouve le locuteur. La plupart du temps, plusieurs critères permettent de classer les pauses, mais parfois les indices sont discordants et la catégorisation ne va pas de soi. On peut alors proposer plusieurs analyses pour un même phénomène.

\section{Synthèse et discussion}

Les mesures acoustiques réalisées sur les pauses silencieuses produites par François Hollande n'ont pas montré de différences significatives, en termes de durée, par rapport aux valeurs généralement retenues dans la littérature (Grosjean, Deschamps, 1975 ; Duez, 2003, par exemple). Par conséquent, il semblerait que l'aspect temporel des pauses ne constitue pas un élément de singularisation majeur de la parole de l'actuel président de la République française, lorsque celui-ci s'exprime dans un débat. Par ailleurs, la catégorisation des pauses selon les critères proposés dans la littérature (voir par exemple Zellner, 1994 ou Ferré, 2003), a permis d'observer une durée généralement plus longue dans les pauses de démarcation que dans les pauses d'hésitation ou de focalisation. Cette durée plus importante est accompagnée par une variabilité accrue qui laisse penser que les pauses démarcatives peuvent parfois cumuler plusieurs valeurs différentes dans le discours politique, ce que confirme d'ailleurs l'analyse du discours en interaction menée en parallèle. Ainsi, les silences en question peuvent à la fois servir à séparer deux séquences sonores distinctes et à focaliser l'attention sur la séquence sonore de droite. En outre, la variabilité relevée sur les pauses de démarcation pourrait également s'expliquer par le fait que c'est principalement en jouant sur ce type de pauses que l'homme ou la femme politique peut donner un autre sens à son discours, en allongeant la durée des silences démarcatifs pour donner une impression de gravité ou en la diminuant pour délivrer une impression de spontanéité, ce que les autres catégories de pauses ne permettent pas de faire. En outre, nous avons également relevé un pourcentage important de pauses d'hésitation et de focalisation, qui pourraient être à l'origine des particularités rythmiques pouvant être observées chez François Hollande. 
Enfin, signalons encore que la comparaison des résultats obtenus pour les deux débats analysés a révélé un débit de parole plus lent chez le candidat victorieux de l'élection présidentielle lors du débat l'opposant à Nicolas Sarkozy. De même, la durée globale des pauses produites par François Hollande était plus importante dans ce face-à-face que dans l'émission qui l'opposait à Martine Aubry. On peut avancer que la solennité du débat face à Nicolas Sarkozy implique que le discours soit plus maîtrisé et que les pauses soient plus marquées.

Quoiqu'il en soit, il nous semble qu'observer comment la pause est utilisée dans la production du discours permet de mieux comprendre de quelle façon le locuteur construit sa parole. Cependant, cette étude rencontre un certain nombre d'obstacles. Si l'approche phonétique permet de relever l'ensemble des pauses dans un discours, de les mesurer et de les décrire objectivement, la phase de catégorisation des différentes occurrences est plus problématique. On a pu mettre en évidence certaines caractéristiques pour définir les différentes pauses, mais il apparaît surtout que leur analyse répond à un travail d'interprétation du chercheur. La classification des différentes pauses ne va pas de soi : elle est construite par l'analyste et doit être justifiée, ce que nous voulions faire ici. Le travail de catégorisation a été mené par différentes individualités afin de pallier les divergences d'appréciation, et les exemples analysés ont été soumis à une étude plus globale du discours au moyen des données vidéo. L'analyse de la pause dans le discours ne peut retenir seulement des éléments acoustiques; l'ensemble du matériau disponible doit être pris en compte : les autres données paraverbales (les intonations, les accentuations), les données discursives environnantes (le cotexte), les données non verbales, le genre du discours (le fait, par exemple, qu'il soit propice ou non à l'interruption peut justifier la présence de pauses démarcatives décalées), la relation interpersonnelle entre les locuteurs ou encore la situation particulière dans laquelle le discours est produit. Pour ce faire, il est important de conjuguer différentes approches pour travailler au mieux la pause discursive, au carrefour de différents domaines de la linguistique.

\section{Références}

BECHET Marion, HIRSCH Fabrice, MARSAC Fabrice, SocK Rudolph, à paraître (2014), «La primaire socialiste. Un événement politique à l'origine d'un nouveau phonostyle?», Neophilologica, $\mathrm{n}^{\circ} 26$.

BOOMER Donald S., 1965, "Hesitation and grammatical encoding», Language and Speech, no8, p. 148-158.

Boomer Donald S., DitTmann Allen T., 1962, «Hesitation pauses and juncture pauses in speech », Language and Speech, n ${ }^{\circ}$, p. 215-220.

CANDEA Maria, 2000, "Contribution à l'étude des pauses silencieuses et des phénomènes dits d' "hésitation" en français oral spontané», thèse de doctorat, Paris 3 Sorbonne nouvelle. 
DISTER Anne, 2008, «L'autocorrection immédiate en français parlé. Le cas des déterminants", Actes des Journées internationales d'analyses statistiques des données textuelles, Lyon, Presses universitaires de Lyon, p. 397-408.

DROMmelL Raimund H., 1980, «Towards a subcategorization of speech pauses», Temporal Variables in Speech, H. W. Dechert, M. Raupach éd., La Haye, Mouton, p. 227-238.

Duez Danielle, 1995, «Perception of hesitations in spontaneous French speech », Proceedings of the XII International Congress of Phonetic Sciences, Stockholm, Royal Institute of Technology, vol.II, p. 498-501.

- 1999, "La fonction symbolique des pauses dans la parole de l'homme politique», Faits de langues, vol. XIII, p. 91-97.

- 2003, "Le pouvoir du silence et le silence du pouvoir. Comment interpréter le discours politique», MediaMorphoses, n॰8, p.77-82.

FERRÉ Gaëlle, 2003, «Les pauses démarcatives déplacées en anglais spontané », Lidil, n०26, p. 155-169.

- 2004, "Les pauses intra-constituants en anglais spontané", Actes des XXVe Journées d'étude sur la parole, Fès, AFCP, p. 217-220.

GoldmAN-EISLER Frieda, 1968, Psycholinguistics. Experiments in Spontaneous Speech, New York, Academic Press.

GrosjeAN François, Deschamps Alain, 1975, «Analyse contrastive des variables temporelles de l'anglais et du français", Phonetica, nº31, p.144-184.

Kerbrat-Orecchion Catherine, 1998 [1990], Les interactions verbales, t. I : Approche interactionnelle et structure des conversations, Paris, Masson et Armand Colin.

- 1998 [1994], Les interactions verbales, t.III : Variations culturelles et échanges rituels, Paris, Armand Colin.

LAROCHE-BOUVY Danielle, 1984, "Les pauses et les silences dans l'interaction verbale », Langage et société, n²29, p. 27-37.

Morel Mary-Annick, Danon-Bolleau Laurent, 1998, Grammaire de l'intonation. L'exemple du français, Paris, Ophrys.

RICHARD Arnaud, SANDrÉ Marion, 2012, «Être (d'accord) ou ne pas être (d'accord)? L'enjeu du débat Aubry-Hollande, entre indépendance et unité», Actes du IIIe Congrès mondial de linguistique française, p. 643-661, en ligne [http://dx.doi. org/10.1051/shsconf/20120100245], consulté le 2 septembre 2013.

- 2013, «Le débat télévisé de primaires. Un nouveau genre médiatique de discours politique en France », Nottingham French Studies, nº52 (2), p. 215-226.

SACKS Harvey, SCHEg LOFf Emanuel A., JefFERSON Gail, 1974, «A simplest systematics for the organization of turn-taking for conversation », Language, no 50 (4), p. 696-735.

SANDRÉ Marion, 2010, "Constances et spécificités des dysfonctionnements interactionnels dans le genre débat politique télévisé. Une application au débat de l'entre-deuxtours de l'élection présidentielle de 2007 ", thèse de doctorat, Université Montpellier 3.

ZeLLnER Brigitte, 1994, "Pauses and the temporal structure of speech », Fundamentals of Speech Synthesis and Speech Recognition, E. Keller éd., Chichester, John Wiley, p. 41-62.

- 1998, «Caractérisation et prédiction du débit de parole en français. Une étude de cas », thèse de doctorat, Université de Lausanne. 